\title{
THE GROUP OF BIHOLOMORPHIC SELF-MAPPINGS OF SCHOTTKY SPACE
}

\author{
Clifford J. Earle
}

\section{Introduction}

The Schottky space $S_{p}$ of marked Schottky groups of genus $p \geq 2$ has very simple embeddings as a domain in $\mathbf{C}^{n}, n=3 p-3$, and is therefore a tempting place to study the Riemann space $R_{p}$ of all closed Riemann surfaces of genus $p$. In fact every closed Riemann surface has many Schottky coverings and every Schottky group has many markings, so $R_{p}$ is the quotient space of $S_{p}$ obtained by considering points in $S_{p}$ to be equivalent if they represent the same Riemann surface. The resulting quotient map from $S_{p}$ to $R_{p}$ is a branched covering, but the covering is not regular. In other words the group of cover transformations, which in this case is the full group Aut $\left(S_{p}\right)$ of biholomorphic self-mappings of $S_{p}$, fails to act transitively on the fibers of the quotient map. Our purpose is to exhibit this non-transitivity very concretely by giving an explicit description of $\operatorname{Aut}\left(S_{p}\right)$. It consists entirely of the familiar mappings induced by changing the marking of the Schottky group.

We state our result formally in Section 3 as Theorem 1. Our proof of Theorem 1 depends on two topological observations, also stated in Section 3 as Theorems 2 and 3. The proofs are given in Sections 4, 5, and 6. They are quite straightforward. The interest of Theorem 1 lies not in the difficulty of its proof but in what it says about $\operatorname{Aut}\left(S_{p}\right)$ : the biholomorphic self-mappings of $S_{p}$ show us which points of $S_{p}$ represent the same Schottky group with different markings, but they do nothing to show which points represent different Schottky coverings of the same Riemann surface.

We state a more concrete version of Theorem 1 in Section 7, where we describe $\operatorname{Aut}\left(S_{p}\right)$ in terms of the obvious action on $S_{p}$ of the outer automorphisms of a free group. Finally, in Section 8 we give explicit formulas for a set of generators of Aut $\left(S_{p}\right)$, using a standard set of global coordinates for $S_{p}$.

Sections 2 and 3 summarize the facts about Schottky space that we need in this paper. More information about Schottky groups can be found in Maskit's book

This research was supported in part by the Institut Mittag-Leffler and by NSF grant DMS8901729 . 
[17] or Bers's paper [3]. Schottky space is a simple example of a quasiconformal deformation space of a Kleinian group. For information about these more general spaces the reader should consult [12], [13], [4], or [16], which is especially relevant to our considerations. The papers [11] and [18] also have some relevance to our work. Our exposition in Section 2 owes something to [11] as well as to [16].

I am indebted to Albert Marden, Gaven Martin, and Bernard Maskit for stimulating discussions about the topology of Schottky coverings. Finally, thanks are due to the people at the Institut Mittag-Leffler for their support and warm hospitality while I was writing this paper.

\section{The Schottky space}

Choose a closed Riemann surface $X$ of genus $p \geq 2$ and a base point $x_{0}$ on $X$. Let $a_{1}, b_{1}, a_{2}, \ldots, b_{p}$ be a standard system of generators for $\pi_{1}\left(X, x_{0}\right)$. This means they are represented by simple loops on $X$ that meet only at $x_{0}$, satisfy the standard relation

$$
\prod_{j=1}^{p} a_{j} b_{j} a_{j}^{-1} b_{j}^{-1}=1,
$$

and are oriented so that $a_{j} \times b_{j}=1$ for each $j$. We denote by $N$ the normal subgroup of $\pi_{1}\left(X, x_{0}\right)$ generated by $b_{1}, b_{2}, \ldots, b_{p}$. The quotient group $\pi_{1}\left(X, x_{0}\right) / N$ is the free group of rank $p$ generated by the images of $a_{1}, \ldots, a_{p}$ under the quotient map.

Let $\Omega \rightarrow X$ be the covering surface of $X$ defined by the subgroup $N$ (see [17]). It is classical that $\Omega$ can be mapped conformally into the complex plane and that any two such embeddings differ only by a Möbius transformation (see Theorem 2D and 19F in Chapter IV of [1]). The group $\pi_{1}\left(X, x_{0}\right) / N$ of cover transformations then becomes a Kleinian group (i.e. a discrete group of Möbius transformations) that acts freely and properly discontinuously on the plane region $\Omega$. We fix such a conformal embedding once and for all, and we denote by $G_{p}$ the Kleinian group of cover transformations. We also choose a system of free generators $g_{1}, \ldots, g_{p}$ for $G_{p}$; they will be used in Section 8 .

The construction above can be applied to any closed Riemann surface of genus $p$, with any standard set of generators for $\pi_{1}$. The resulting covering surfaces are called Schottky coverings, and the associated Kleinian groups are the Schottky groups of genus $p$. By definition, a marked Schottky group of genus $p$ is an isomorphism $\theta: G_{p} \rightarrow G$ of our distinguished Schottky group $G_{p}$ onto some Schottky group $G$. Two marked Schottky groups $\theta$ and $\theta^{\prime}$ are equivalent if and only if there is a Möbius transformation $A$ such that

$$
\theta^{\prime}(g)=A \theta(g) A^{-1} \quad \text { for every } g \text { in } G_{p} .
$$

The set of equivalence classes $[\theta]$ is the Schottky space $S_{p}$. It is clear that the space $S_{p}$ does not depend in an essential way on our choice of $G_{p}$, for the choice of 
an isomorphism $\theta: G_{p} \rightarrow G$ amounts to the same thing as the choice of a system of free generators $\theta\left(g_{1}\right), \ldots, \theta\left(g_{p}\right)$ for the Schottky group $G$.

A remarkable observation of Chuckrow [6] says that for any marked Schottky group $\theta: G_{p} \rightarrow G$ there is a quasiconformal homeomorphism $f$ of the Riemann sphere such that $\theta(g)=f g f^{-1}$ for every $g$ in $G_{p}$. Therefore $S_{p}$ coincides with the quasiconformal deformation space (see [12] or [16]) of $G_{p}$, and the theory of these deformation spaces applies to $S_{p}$. In particular, $S_{p}$ is a complex manifold of dimension $n=3 p-3$, and any injective holomorphic map of $S_{p}$ into $\mathbf{C}^{n}$ is a biholomorphic map onto a region in $\mathbf{C}^{n}$. Such injections are easily defined in terms of fixed points and multipliers of the transformations $\theta(g)$. See for example [3], [11], [13], or Section 8.

\section{The theorems}

A fundamental theorem of Maskit (see Corollary 8 in [16]) states that the universal covering space of $S_{p}$ is the Teichmüller space $T_{p}$ and identifies the group of cover transformations. (Bers [2] had also proved that $T_{p}$ covers $S_{p}$, but his description of the cover transformations was not explicit.) We shall describe that group presently. First we review the definition and some properties of $T_{p}$.

Let $\mathcal{M}(X)$ be the space of all smooth (class $C^{\infty}$ ) conformal structures on $X$, with the usual topology of $C^{\infty}$ convergence (see [7], [8], or [18]). The group $\operatorname{Diff}^{+}(X)$ of all sense-preserving smooth diffeomorphisms of $X$ acts (from the right) on $\mathcal{M}(X)$ by pullback. The space $T_{p}$ is the quotient space

$$
T_{p}=\mathcal{M}(X) / \operatorname{Diff}_{0}(X)
$$

of $\mathcal{M}(X)$ by the normal subgroup $\operatorname{Diff}_{0}(X)$, which consists of the diffeomorphisms that are homotopic to the identity. $T_{p}$ inherits a complex analytic structure from $\mathcal{M}(X)$ and is a contractible complex manifold of dimension $3 p-3$, homeomorphic to $\mathbf{C}^{3 p-3}$. The quotient group $\operatorname{Mod}(X)=\operatorname{Diff}^{+}(X) / \operatorname{Diff}_{0}(X)$ acts properly discontinuously on $T_{p}$ as a group of biholomorphic maps, and a deep theorem of Royden says that every biholomorphic self-mapping of $T_{p}$ is induced by some member of $\operatorname{Mod}(X)$. All this is classical (see [7], [9], [12], or [19]).

Now recall from Section 2 the normal subgroup $N$ of $\pi_{1}\left(X, x_{0}\right)$, which determines the Schottky covering surface $\Omega \rightarrow X$. Following Maskit, we introduce some subgroups of $\operatorname{Diff}^{+}(X)$ and $\operatorname{Mod}(X)$. Let $\operatorname{Diff}^{+}(X, N)$ be the group of all $f$ in $\operatorname{Diff}^{+}(X)$ that can be lifted to a diffeomorphism $\tilde{f}: \Omega \rightarrow \Omega$, and let Diff $_{0}(X, N)$ be the subgroup consisting of all $f$ that can be lifted to a diffeomorphism $\tilde{f}: \Omega \rightarrow \Omega$ that commutes with the group $G_{p}$.

Since $\operatorname{Diff}_{0}(X)$ is a subgroup of $\operatorname{Diff}_{0}(X, N)$, we can form the quotient groups

$$
\begin{aligned}
& \operatorname{Mod}^{*}(X, N)=\operatorname{Diff}^{+}(X, N) / \operatorname{Diff}_{0}(X), \\
& \operatorname{Mod}_{*}(X, N)=\operatorname{Diff}_{0}(X, N) / \operatorname{Diff}_{0}(X) .
\end{aligned}
$$

These are subgroups of $\operatorname{Mod}(X)$, so they act properly discontinuously on $T_{p}$. Maskit proved 
Theorem A (Maskit [16]). The group $\operatorname{Mod}_{*}(X, N)$ acts freely on $T_{p}$, and $S_{p}$ equals the quotient space $T_{p} / \operatorname{Mod}_{*}(X, N)$.

$\operatorname{Diff}_{0}(X, N)$ is a normal subgroup of $\operatorname{Diff}^{+}(X, N)$, so the quotient group

$$
\Gamma_{p}=\operatorname{Diff}^{+}(X, N) / \operatorname{Diff}_{0}(X, N)=\operatorname{Mod}^{*}(X, N) / \operatorname{Mod}_{*}(X, N)
$$

acts on $S_{p}$ as a group of biholomorphic self-mappings. Our main result is

Theorem 1. Every biholomorphic self-mapping of $S_{p}$ is induced by some member of $\Gamma_{p}$.

As J.A. Gentilesco pointed out under more general circumstances (see Theorem VIII of [10]), Theorem 1 is an easy consequence of Royden's analogous theorem about $T_{p}$ and the purely topological

Theorem 2. The normalizer of $\operatorname{Mod}_{*}(X, N)$ in $\operatorname{Mod}(X)$ is $\operatorname{Mod}^{*}(X, N)$.

We deduce Theorem 2 from the following topological theorem, which provides a tight relationship between the groups $N$ and $\operatorname{Mod}_{*}(X, N)$.

Theorem 3. Let $c$ in $\pi_{1}\left(X, x_{0}\right)$ be represented by a simple loop. Then $c \in N$ if and only if the Dehn twist $\tau(c)$ on $c$ belongs to $\operatorname{Mod}_{*}(X, N)$.

Remarks. 1) For a discussion of Dehn twists see [5].

2) Theorems 1 and 2 measure the failure of $\operatorname{Aut}\left(S_{p}\right)$ to act transitively on the fibers of the quotient map from $S_{p}$ to $R_{p}$. The Riemann space is the quotient

$$
R_{p}=T_{p} / \operatorname{Mod}(X)
$$

and $S_{p} / \operatorname{Aut}\left(S_{p}\right)=T_{p} / \operatorname{Mod}^{*}(X, N)$, so the map from $S_{p} / \operatorname{Aut}\left(S_{p}\right)$ to $R_{p}$ has fibers generically isomorphic to the set of cosets of $\operatorname{Mod}^{*}(X, N)$ in $\operatorname{Mod}(X)$.

It is known that $\operatorname{Mod}^{*}(X, N)$ is a rather thin subgroup of $\operatorname{Mod}(X)$. In fact Masur [18] showed that $\operatorname{Mod}^{*}(X, N)$ acts properly discontinuously on a nontrivial open subset of Thurston's sphere $P \mathcal{F}$ of projective measured foliations. In contrast, $\operatorname{Mod}(X)$ acts minimally, even ergodically, on $P \mathcal{F}$ (see[18]).

The Dehn twist $\tau\left(a_{1}\right)$ provides an obvious example of an element of $\operatorname{Mod}(X)$ that does not belong to $\operatorname{Mod}^{*}(X, N)$.

3) The action of the Schottky group $G_{p}$ extends to hyperbolic 3 -space, and the quotient of hyperbolic 3 -space by $G_{p}$ is a solid handlebody $H$ bounded by the surface $X$. Diff ${ }^{+}(X, N)$ is just the subgroup of $\operatorname{Diff}^{+}(X)$ that can be extended to $H$, and Diff ${ }_{0}(X, N)$ consists of the diffeomorphisms whose extensions to $H$ are homotopic to the identity in $H$.

4) According to an interesting theorem of Luft [14], the Dehn twists $\tau(c)$ in Theorem 3 generate the group $\operatorname{Mod}_{*}(X, N)$.

5) Hejhal [11] characterizes the covering group $\operatorname{Mod}_{*}(X, N)$ by a lifting property that differs slightly from (3.2). Maskit's characterization in terms of (3.2) is more useful for us here. 


\section{Proof of Theorem 1}

For the reader's convenience we shall derive Theorem 1 from Theorem 2, following the method of Gentilesco [10]. Let $\varphi: S_{p} \rightarrow S_{p}$ be a biholomorphic self-mapping of $S_{p}$. The quotient map from $T_{p}$ to $S_{p}$ in Maskit's Theorem A is a holomorphic universal covering, so $\varphi$ lifts to a biholomorphic self-mapping $\psi$ of $T_{p}$. By Royden's theorem, $\psi$ is induced by an element $\theta$ of $\operatorname{Mod}(X)$. Since $\theta$ and $\theta^{-1}$ both induce the maps on $S_{p}$, we must have $\theta \sigma \theta^{-1} \in \operatorname{Mod}_{*}(X, N)$ and $\theta^{-1} \sigma \theta \in \operatorname{Mod}_{*}(X, N)$ for all $\sigma$ in $\operatorname{Mod}_{*}(X, N)$. Therefore $\theta$ belongs to the normalizer of $\operatorname{Mod}_{*}(X, N)$, which, by Theorem 2, equals $\operatorname{Mod}^{*}(X, N)$. QED

\section{Proof of Theorem 2}

Since $\operatorname{Diff}_{0}(X) \subset \operatorname{Diff}_{0}(X, N)$, Theorem 2 is equivalent to the statement that the normalizer of $\operatorname{Diff}_{0}(X, N)$ in $\operatorname{Diff}^{+}(X)$ is $\operatorname{Diff}^{+}(X, N)$. That is what we shall prove.

Let $f$ belong to $\operatorname{Diff}^{+}(X)$ and let $c$ belong to $\pi_{1}\left(X, x_{0}\right)$. Since $N$ is a normal subgroup, the statement that $f(c)$ belongs to $N$ makes sense even though $f$ need not preserve the base point $x_{0}$. Covering space theory then tells us that $f \in \operatorname{Diff}^{+}(X, N)$ if and only if both $f(c) \in N$ and $f^{-1}(c) \in N$ whenever $c \in N$. It obviously suffices to have $f\left(b_{j}\right) \in N$ and $f^{-1}\left(b_{j}\right) \in N$ for $1 \leq j \leq p$.

Now let $f$ belong to the normalizer of $\operatorname{Diff}_{0}(X, N)$. Let $\tau\left(b_{j}\right)$ be (a representative in $\operatorname{Diff}^{+}(X)$ of $)$ the Dehn twist on $b_{j}$. By Theorem $3, \tau\left(b_{j}\right) \in \operatorname{Diff}_{0}(X, N)$, so $f \tau\left(b_{j}\right) f^{-1} \in \operatorname{Diff}_{0}(X, N)$. But $f \tau\left(b_{j}\right) f^{-1}$ is (represents) the Dehn twist on $f\left(b_{j}\right)$ (see [5]). Therefore, by Theorem $3, f\left(b_{j}\right) \in N$. The same reasoning applied to $f^{-1}$ shows that $f^{-1}\left(b_{j}\right)$ also belongs to $N$. Therefore $f \in \operatorname{Diff}^{+}(X, N)$. QED

\section{Proof of Theorem 3}

We will do the trivial implication first. Suppose the simple geodesic loop $\gamma$ represents $c$ in $N$. Choose a small collar $C$ about $\gamma$. By definition of the covering surface $\pi: \Omega \rightarrow X$, each connected component of $\pi^{-1}(C)$ in $\Omega$ is mapped homeomorphically onto $C$ by $\pi$.

Now choose a diffeomorphism $f$ that equals the identity in $X \backslash C$ and represents the Dehn twist $\tau(c)$. Lift $f$ to a diffeomorphism $\tilde{f}: \Omega \rightarrow \Omega$ by putting $\tilde{f}=\mathrm{id}$ in $\Omega \backslash \pi^{-1}(C)$ and $\tilde{f}=(\pi \mid \tilde{C})^{-1} \circ f \circ(\pi \mid \tilde{C})$ in each connected component $\tilde{C}$ of $\pi^{-1}(C)$. Since $\tilde{f}$ commutes with $G_{p}, f \in \operatorname{Diff}_{0}(X, N)$ and $\tau(c) \in \operatorname{Mod}_{*}(X, N)$ as required.

Conversely, suppose $\tau(c) \in \operatorname{Mod}_{*}(X, N)$. As before, we choose a small collar $C$ about a simple geodesic loop $\gamma$ that represents $c$, and we represent $\tau(c)$ by a diffeomorphism $f$ that equals the identity in $X \backslash C$. By hypothesis, $f$ has a lift $\tilde{f}: \Omega \rightarrow \Omega$ that commutes with the group $G_{p}$.

We shall assume that $c \notin N$ and look for a contradiction. Let $\beta_{1}$ and $\beta_{2}$ be the two boundary loops of $C$. Since $c \notin N$, each connected component of 
$\pi^{-1}\left(\beta_{1} \cup \beta_{2}\right)$ is a simple arc $\beta$ in $\Omega$. Let $\varphi_{\beta}$ be a generator of the infinite cyclic group of all $\varphi$ in $G_{p}$ such that $\varphi(\beta)=\beta$. Then $\beta$ connects the two fixed points of $\varphi_{\beta}$ (which are boundary points of $\Omega$ ).

First we shall prove that $\tilde{f}=$ id in $\Omega \backslash \pi^{-1}(C)$. Let $Y$ be a connected component of $\Omega \backslash \pi^{-1}(C)$, and let $H$ be the subgroup of $G_{p}$ that maps $Y$ onto itself. Since $f=$ id on $X \backslash C$, there is some $\psi$ in $G_{p}$ such that $\tilde{f}=\psi$ on $Y$. Since $\tilde{f}$ commutes with $G_{p}, \psi$ commutes with the subgroup $H$. Suppose $\psi \neq i d$. Then $H$ is cyclic and all nontrivial elements of $H$ have the same two fixed points. Now the boundary of $Y$ in $\Omega$ consists of $\operatorname{arcs} \beta$ in $\pi^{-1}\left(\beta_{1} \cup \beta_{2}\right)$. Each $\varphi_{\beta}$ belongs to $H$, and $\beta$ connects its fixed points, so $Y$ must be a Jordan region bounded by the union of two $\operatorname{arcs} \beta, \beta^{\prime}$ and their common endpoints. Therefore $Y / H$ is an annulus. Since $Y / H$ is a connected component of $X \backslash C$ and $X$ has genus $p \geq 2$, this is nonsense. Therefore $\psi=\mathrm{id}$, so $\tilde{f}$ is the identity in $Y$ and hence in $\Omega \backslash \pi^{-1}(C)$.

It is now easy to reach the desired contradiction. Let $\tilde{C}$ be a connected component of $\pi^{-1}(C)$. Then $\tilde{C}$ is a Jordan domain whose boundary is the union of two $\operatorname{arcs} \beta$ and $\beta^{\prime}$ as above, and their common endpoints. The stabilizer of $\tilde{C}$ in $G_{p}$ is the cyclic group generated by $\varphi_{\beta}$. Since $\tilde{f}=$ id in $\Omega \backslash \pi^{-1}(C), \tilde{f}$ maps $\tilde{C}$ onto itself and equals the identity on the boundary of $\tilde{C}$. In addition, $\tilde{f}$ commutes with $\varphi_{\beta}$.

It is easy to construct a $\varphi_{\beta}$-equivariant homotopy of $\tilde{f}$ to the identity in $\tilde{C}$, holding the boundary of $\tilde{C}$ pointwise fixed. (For instance there is a conformal map that takes $\tilde{C}$ to a closed horizontal strip $\{z=x+i y ;|y| \leq r\}$ and $\varphi_{\beta}$ to $z \mapsto z+1$. We can then set $\tilde{f}_{t}(z)=t z+(1-t) \tilde{f}(z)$ in the closed strip.) Projecting that homotopy to the collar $C$ we find that $f$ is homotopic to the identity in $X$, contradicting the fact that $f$ represents $\tau(c)$. This contradiction implies that $c \in N$. QED

\section{The action of the outer automorphism group}

Let $\operatorname{Aut}\left(G_{p}\right)$ be the group of all automorphisms of the group $G_{p}$, and let $\operatorname{Inn}\left(G_{p}\right)$ be the normal subgroup of inner automorphisms. Aut $\left(G_{p}\right)$ acts in an obvious way on the set of marked Schottky groups: if $\theta: G_{p} \rightarrow G$ is a marked Schottky group and $\alpha \in \operatorname{Aut}\left(G_{p}\right)$, then $\theta \cdot \alpha$ is the marked Schottky group $\theta$ 。 $\alpha: G_{p} \rightarrow G$. This action obviously preserves equivalence classes and induces the action

$$
[\theta] \cdot \alpha=[\theta \circ \alpha] \quad \text { if }[\theta] \in S_{p} \quad \text { and } \alpha \in \operatorname{Aut}\left(G_{p}\right)
$$

of $\operatorname{Aut}\left(G_{p}\right)$ on $S_{p}$. The subgroup $\operatorname{Inn}\left(G_{p}\right)$ acts trivially on $S_{p}$, so (7.1) defines an action of the outer automorphism group

$$
\text { Outer } \operatorname{Aut}\left(G_{p}\right)=\operatorname{Aut}\left(G_{p}\right) / \operatorname{Inn}\left(G_{p}\right)
$$

on $S_{p}$. In terms of these actions Theorem 1 takes the concrete and explicit form 
Theorem 1'. For each $\alpha$ in Aut $\left(G_{p}\right)$ the self-mapping of $S_{p}$ defined by (7.1) is biholomorphic. Every biholomorphic self-mapping of $S_{p}$ has the form (7.1) for some $\alpha$.

The proof is simply a matter of being explicit about the action of $\Gamma_{p}$ in Theorem 1. First we must describe the standard map of $\mathcal{M}(X)$ onto $S_{p}$. Each $\mu$ in $\mathcal{M}(X)$ defines a new Riemann surface structure on $X$, which determines a new $G_{p}$-invariant Riemann surface structure on $\Omega$ via the covering map $\pi: \Omega \rightarrow X$. We denote the resulting Riemann surfaces by $\Omega^{\mu}$ and $X^{\mu}$. Since $\pi: \Omega^{\mu} \rightarrow X^{\mu}$ is a Schottky covering there is a conformal mapping $w^{\mu}$ of $\Omega^{\mu}$ into the complex plane. The group $G^{\mu}=w^{\mu} G_{p}\left(w^{\mu}\right)^{-1}$ is a Schottky group, and the isomorphism

$$
g \mapsto \theta^{\mu}(g)=\left(w^{\mu}\right) \circ g \circ\left(w^{\mu}\right)^{-1} \quad \text { if } \quad g \in G
$$

defines a marked Schottky group. Its equivalence class $\left[\theta^{\mu}\right]$ depends only on $\mu$ because the conformal map $w^{\mu}$ is unique up to composition with a Möbius transformation. The map $\mu \mapsto\left[\theta^{\mu}\right]$ from $\mathcal{M}(X)$ to $S_{p}$ factors through $T_{p}$ (= $\left.\mathcal{M}(X) / \operatorname{Diff}_{0}(X)\right)$ to produce Maskit's universal covering map (see [12] or [16]).

Recall that $\operatorname{Diff}^{+}(X)$ acts on $\mathcal{M}(X)$ by pullback: the map $f: X^{\mu \cdot f} \rightarrow X^{\mu}$ is conformal for every $f$ in $\operatorname{Diff}^{+}(X)$ and $\mu$ in $\mathcal{M}(X)$. The subgroup $\operatorname{Diff}^{+}(X, N)$ acts on $S_{p}$ by

$$
\left[\theta^{\mu}\right] \cdot f=\left[\theta^{\mu \cdot f}\right] \quad \text { if } \quad\left[\theta^{\mu}\right] \in S_{p} \quad \text { and } \quad f \in \operatorname{Diff}^{+}(X, N) .
$$

The normal subgroup $\operatorname{Diff}_{0}(X, N)$ acts trivially, and (7.3) induces the action of the quotient group $\Gamma_{p}$ in Theorem 1.

According to Theorem 1 every biholomorphic self-mapping of $S_{p}$ has the form (7.3). To compute $\left[\theta^{\mu \cdot f}\right]$ for $f$ in $\operatorname{Diff}^{+}(X, N)$ we choose a lift $\tilde{f}: \Omega \rightarrow \Omega$ and observe that $\tilde{f}: \Omega^{\mu \cdot f} \rightarrow \Omega^{\mu}$ is conformal. Therefore $w^{\mu} \circ \tilde{f}$ maps $\Omega^{\mu \cdot f}$ conformally into the complex plane, so

$$
\theta^{\mu \cdot f}(g)=w^{\mu} \circ\left(\tilde{f} \circ g \circ \tilde{f}^{-1}\right) \circ\left(w^{\mu}\right)^{-1}=\theta^{\mu}\left(\tilde{f} \circ g \circ \tilde{f}^{-1}\right)
$$

for all $g$ in $G$. Thus (7.3) takes the form

$$
\left[\theta^{\mu}\right] \cdot f=\left[\theta^{\mu} \circ \alpha_{\tilde{f}}\right] \quad \text { if } \quad\left[\theta^{\mu}\right] \in S_{p} \quad \text { and } \quad f \in \operatorname{Diff}_{+}(X, N),
$$

where $\alpha_{\tilde{f}}$ in $\operatorname{Aut}\left(G_{p}\right)$ is the automorphism $g \mapsto \tilde{f} \circ g \circ \tilde{f}^{-1}$. We see that every biholomorphic self-mapping of $S_{p}$ is indeed of the form (7.1).

To verify that all the maps (7.1) are biholomorphic we must show that every $\alpha$ in $\operatorname{Aut}\left(G_{p}\right)$ is of the form $\alpha_{\tilde{f}}$. That is the content of Chuckrow's observation (Theorem 2 of [6]), which for any given $\alpha$ guarantees the existence of a sensepreserving diffeomorphism $\tilde{f}: \Omega \rightarrow \Omega$ such that $\alpha(g)=\tilde{f} \circ g \circ \tilde{f}^{-1}$ for all $g$ in $G_{p}$. The map $\tilde{f}$ covers a diffeomorphism $f: X \rightarrow X$. By definition, $f \in \operatorname{Diff}^{+}(X, N)$ and $\alpha=\alpha_{\tilde{f}}$. The proof is complete. 
Remarks. 1) The outer automorphism $\left[\alpha_{\tilde{f}}\right]$ depends only on $f$, and the map $f \mapsto\left[\alpha_{\tilde{f}}\right]$ induces an isomorphism between the groups $\Gamma_{p}$ and $\operatorname{Outer} \operatorname{Aut}\left(G_{p}\right)$.

2) It is a striking fact that every outer automorphism of $G_{p}$ is induced by a sense-preserving diffeomorphism of $X$ and that sense-reversing diffeomorphisms are not required. The geometric reason for this is that there is a sense-reversing diffeomorphism $\tilde{f}: \Omega \rightarrow \Omega$ that commutes with the group $G_{p}$. This is easy to see if we take $G_{p}$ to be a Fuchsian group of the second kind and $\tilde{f}$ to be inversion in the fixed circle.

\section{Explicit formulas}

Finally, we shall borrow a set of global coordinates for $S_{p}$ from Hejhal [11] and give formulas in these coordinates for a set of generators of $\operatorname{Aut}\left(S_{p}\right)$. Following [11], for any marked Schottky group $\theta: G_{p} \rightarrow G$ we put $L_{j}=\theta\left(g_{j}\right)$ for $1 \leq j \leq p$ and we set $a_{j}, b_{j}$, and $\lambda_{j}$ equal to the attracting fixed point, repelling fixed point, and multiplier of $L_{j}$, defining the multiplier so that $0<\left|\lambda_{j}\right|<1$. Replacing $\theta$ by an equivalent isomorphism, we can normalize the $p$-tuple $\left(L_{1}, \ldots, L_{p}\right)$ so that $a_{1}=0, a_{2}=1$, and $b_{1}=\infty$. The injective holomorphic map

$$
[\theta] \mapsto\left(a_{3}, \ldots, a_{p}, b_{2}, \ldots, b_{p}, \lambda_{1}, \ldots, \lambda_{p}\right) \in \mathbf{C}^{3 p-3}
$$

then defines a global coordinate system for $S_{p}$ (see [11] and [13]), allowing us to interpret $S_{p}$ as a region in $\mathrm{C}^{3 p-3}$ and $\operatorname{Aut}\left(S_{p}\right)$ as the group of biholomorphic self-mappings of that region.

Now every member of $\operatorname{Aut}\left(S_{p}\right)$ is induced by an automorphism of the free group $G_{p}$, and a theorem of Nielsen (see Corollary N1 in Section 3.5 of [15]) implies that $\operatorname{Aut}\left(G_{p}\right)$ is generated by these four automorphisms:

$$
\begin{gathered}
\alpha_{1}\left(g_{1}\right)=g_{p}, \quad \text { and } \quad \alpha_{1}\left(g_{j}\right)=g_{j-1} \quad \text { if } j>1, \\
\alpha_{2}\left(g_{1}\right)=g_{2}, \quad \alpha_{2}\left(g_{2}\right)=g_{1}, \quad \text { and } \quad \alpha_{2}\left(g_{j}\right)=g_{j} \quad \text { if } j>2, \\
\alpha_{3}\left(g_{1}\right)=g_{1}^{-1}, \quad \text { and } \quad \alpha_{3}\left(g_{j}\right)=g_{j} \quad \text { if } j>1, \\
\alpha_{4}\left(g_{1}\right)=g_{1}, \quad \alpha_{4}\left(g_{2}\right)=g_{2}^{-1} g_{1}, \quad \text { and } \quad \alpha_{4}\left(g_{j}\right)=g_{j} \quad \text { if } j>2 .
\end{gathered}
$$

We must calculate their effect on the region $S_{p}$.

The automorphism $\alpha_{1}$ transforms $\left(L_{1}, \ldots, L_{p}\right)$ to $\left(L_{p}, L_{1}, \ldots, L_{p-1}\right)$. Conjugation by

$$
T(z)=\frac{b_{p}\left(z-a_{p}\right)}{a_{p}\left(z-b_{p}\right)}
$$


produces the normalized $p$-tuple

$$
\left(T L_{p} T^{-1}, T L_{1} T^{-1}, \ldots, T L_{p-1} T^{-1}\right)
$$

and replaces each fixed point by its image under $T$, so $\alpha_{1}$ induces the map

$$
(a, b, \lambda) \mapsto\left(T\left(a_{2}\right), \ldots, T\left(a_{p-1}\right), T\left(b_{1}\right), \ldots, T\left(b_{p-1}\right), \lambda_{p}, \lambda_{1}, \ldots, \lambda_{p-1}\right) .
$$

(We use $(a, b, \lambda)$ as an abbreviation for $\left(a_{3}, \ldots, a_{p}, b_{2}, \ldots, b_{p}, \lambda_{1}, \ldots, \lambda_{p}\right)$, and we remind the reader that $a_{2}=1$ and $b_{1}=\infty$.)

The automorphism $\alpha_{2}$ produces the $p$-tuple $\left(L_{2}, L_{1}, L_{3}, \ldots, L_{p}\right)$, which is conjugated to normalized form by the transformation

$$
S(z)=\frac{b_{2}(z-1)}{z-b_{2}} .
$$

Therefore $\alpha_{2}$ induces the map

$$
(a, b, \lambda) \mapsto\left(S\left(a_{3}\right), \ldots, S\left(a_{p}\right), S\left(b_{1}\right), S\left(b_{3}\right), \ldots, S\left(b_{p}\right), \lambda_{2}, \lambda_{1}, \lambda_{3}, \ldots, \lambda_{p}\right) .
$$

The automorphism $\alpha_{3}$ leads to $\left(L_{1}^{-1}, L_{2}, L_{3}, \ldots, L_{p}\right)$. The transformation $L_{1}^{-1}$ has multiplier $\lambda_{1}$, attracting fixed point $\infty$, and repelling fixed point 0 . Conjugation by $z \mapsto 1 / z$ produces a normalized $p$-tuple, so $\alpha_{3}$ induces the map

$$
(a, b, \lambda) \mapsto\left(\frac{1}{a_{3}}, \ldots, \frac{1}{a_{p}}, \frac{1}{b_{2}}, \ldots, \frac{1}{b_{p}}, \lambda_{1}, \ldots, \lambda_{p}\right) .
$$

All this was very easy, but $\alpha_{4}$ requires more effort. We must normalize the $p$-tuple $\left(L_{1}, L_{2}^{-1} L_{1}, L_{3}, \ldots, L_{p}\right)$, so we need to find $a^{*}, b^{*}$, and $\lambda^{*}$, the attracting fixed point, repelling fixed point, and multiplier of $L_{2}^{-1} L_{1}$. We know that $L_{1}(z)=$ $\lambda_{1} z$ and that $L_{2}$ is represented by the matrix

$$
\left(\begin{array}{cc}
1-b_{2} \lambda_{2} & b_{2}\left(\lambda_{2}-1\right) \\
1-\lambda_{2} & \lambda_{2}-b_{2}
\end{array}\right)
$$

in $G L(2, \mathbf{C})$. The matrix

$$
A=\left(\begin{array}{cc}
\lambda_{1}\left(\lambda_{2}-b_{2}\right) & b_{2}\left(1-\lambda_{2}\right) \\
\lambda_{1}\left(\lambda_{2}-1\right) & 1-b_{2} \lambda_{2}
\end{array}\right)
$$

then represents $L_{2}^{-1} L_{1}$. (Our matrices do not necessarily have determinant one.) The multiplier $\lambda^{*}$ of $L_{2}^{-1} L_{1}$ satisfies

$$
\lambda^{*}+\left(\lambda^{*}\right)^{-1}+2=\operatorname{trace}(A)^{2} / \operatorname{det}(A),
$$


so it is a solution of the quadratic equation

$$
\lambda_{1} \lambda_{2}\left(b_{2}-1\right)^{2}\left[\left(\lambda^{*}\right)^{2}+1\right]=\left[\lambda_{1}^{2}\left(\lambda_{2}-b_{2}\right)^{2}+\left(1-b_{2} \lambda_{2}\right)^{2}-2 b_{2} \lambda_{1}\left(\lambda_{2}-1\right)^{2}\right] \lambda^{*}
$$

In fact $\lambda^{*}$ is the unique solution of (8.8) satisfying $\left|\lambda^{*}\right|<1$. Thus $\lambda^{*}$ is an algebraic, but not a rational, function of $\lambda_{1}, \lambda_{2}$, and $b_{2}$.

Since $L_{2}^{-1} L_{1}$ is represented by the matrix $A$ in (8.7), the product $a^{*} b^{*}$ of its fixed points equals $b_{2} / \lambda_{1}$. Put $\zeta=\lambda_{1} b^{*}$. Then $\zeta a^{*}-b_{2}=\zeta-\lambda_{1} b^{*}=0$, so the matrix

$$
\begin{aligned}
B & =\left(\begin{array}{cc}
\zeta & b_{2} \\
\lambda_{1} & \zeta
\end{array}\right)\left(\begin{array}{cc}
\lambda^{*} & 0 \\
0 & 1
\end{array}\right)\left(\begin{array}{cc}
\zeta & -b_{2} \\
-\lambda_{1} & \zeta
\end{array}\right) \\
& =\left(\begin{array}{ll}
\lambda^{*} \zeta^{2}-b_{2} \lambda_{1} & b_{2}\left(1-\lambda^{*}\right) \zeta \\
\lambda_{1}\left(\lambda^{*}-1\right) \zeta & \zeta^{2}-b_{2} \lambda_{1} \lambda^{*}
\end{array}\right)
\end{aligned}
$$

also represents $L_{2}^{-1} L_{1}$.

Now the equation $\left(\lambda_{2}-1\right) B=\left(\lambda^{*}-1\right) \zeta A$ yields a pair of quadratic equations for $\zeta$. Eliminating $\zeta^{2}$ between them we find that

$$
\begin{gathered}
\zeta=\frac{b_{2} \lambda_{1}\left(1-\lambda_{2}\right)\left(\lambda^{*}+1\right)}{\left(1-b_{2} \lambda_{2}\right) \lambda^{*}-\lambda_{1}\left(\lambda_{2}-b_{2}\right)} \\
a^{*}=\frac{\left(1-b_{2} \lambda_{2}\right) \lambda^{*}-\lambda_{1}\left(\lambda_{2}-b_{2}\right)}{\lambda_{1}\left(1-\lambda_{2}\right)\left(\lambda^{*}+1\right)}
\end{gathered}
$$

and

$$
\frac{b^{*}}{a^{*}}=\frac{\lambda_{1}\left(b_{2}-\lambda_{2}\right) \lambda^{*}+\left(1-b_{2} \lambda_{2}\right)}{\left(1-b_{2} \lambda_{2}\right) \lambda^{*}+\lambda_{1}\left(b_{2}-\lambda_{2}\right)}
$$

(To obtain (8.10) we use (8.8) to simplify the right hand side of the equation $b^{*} / a^{*}=\zeta^{2} / b_{2} \lambda_{1}$.) The map induced by $\alpha_{4}$ is therefore

$$
(a, b, \lambda) \mapsto\left(\frac{a_{3}}{a^{*}}, \ldots, \frac{a_{p}}{a^{*}}, \frac{b^{*}}{a^{*}}, \frac{b_{3}}{a^{*}}, \ldots, \frac{b_{p}}{a^{*}}, \lambda_{1}, \lambda^{*}, \lambda_{3}, \ldots, \lambda_{p}\right)
$$

where $\lambda^{*}$ is the unique solution of (8.8) with $\left|\lambda^{*}\right|<1, a^{*}$ is given by (8.9), and $b^{*} / a^{*}$ by $(8.10)$.

We sum up our results in a final

Proposition. The group of all biholomorphic self-mappings of the region $S_{p}$ in $\mathbf{C}^{3 p-3}$ is generated by the four transformations $\left(8.1^{\prime}\right)$ through $\left(8.4^{\prime}\right)$. 
Remark. The special case $p=2$ has particular interest, both because Kleinian groups with two generators have been much studied and because the results become simpler and even more explicit. Notice first that the automorphisms $\alpha_{1}$ and $\alpha_{2}$ coincide when $p=2$, so $\alpha_{2}, \alpha_{3}$, and $\alpha_{4}$ generate $\operatorname{Aut}\left(G_{2}\right)$. Secondly, another theorem of Nielsen (see Corollary N4 in Section 3.5 of [15]) tells us that Outer $\operatorname{Aut}\left(G_{2}\right)$ is canonically isomorphic to the automorphism group of the abelianization of $G_{2}$, so Outer Aut $\left(G_{2}\right)$ is isomorphic to the group $G L(2, Z)$ of two-by-two unimodular integer matrices. Finally, since every closed Riemann surface of genus two is hyperelliptic, the automorphism of $G_{2}$ defined by $g_{j} \mapsto g_{j}^{-1}$, $j=1$ or 2 , acts trivially on the Schottky space $S_{2}$. Therefore the group of biholomorphic self-mappings of $S_{2}$ is isomorphic to

$$
P G L(2, Z)=G L(2, Z) /\{ \pm I\}
$$

The generating matrices

$$
\pm\left(\begin{array}{ll}
0 & 1 \\
1 & 0
\end{array}\right), \quad \pm\left(\begin{array}{cc}
-1 & 0 \\
0 & 1
\end{array}\right), \quad \text { and } \quad \pm\left(\begin{array}{cc}
1 & 1 \\
0 & -1
\end{array}\right)
$$

correspond to the self-mappings

$$
\left(b_{2}, \lambda_{1}, \lambda_{2}\right) \mapsto\left(b_{2}, \lambda_{2}, \lambda_{1}\right), \quad\left(b_{2}, \lambda_{1}, \lambda_{2}\right) \mapsto\left(\frac{1}{b_{2}}, \lambda_{1}, \lambda_{2}\right),
$$

and

$$
\left(b_{2}, \lambda_{1}, \lambda_{2}\right) \mapsto\left(\frac{b^{*}}{a^{*}}, \lambda_{1}, \lambda^{*}\right)
$$

respectively, with $\lambda^{*}$ and $b^{*} / a^{*}$ determined as in $\left(8.4^{\prime}\right)$.

\section{References}

[1] Ahlfors, L.V., and L. SARIo: Riemann surfaces. - Princeton University Press, Princeton, New Jersey, 1960.

[2] Bers, L.: Spaces of Kleinian groups. - In Several complex variables, Maryland, 1970. Lecture Notes in Mathematics 155. Springer-Verlag, Berlin-Heidelberg-New York, 1970, 9-34.

[3] Bers, L.: Automorphic forms for Schottky groups. - Adv. in Math. 16, 1975, 332-361.

[4] Bers, L., and I. KRA (eds.): A crash course on Kleinian groups. - Lecture Notes in Mathematics 400. Springer-Verlag, Berlin-Heidelberg-New York, 1974.

[5] Birman, J.S.: The algebraic structure of surface mapping class groups. - In Discrete groups and automorphic functions, edited by W.J. Harvey, Academic Press, London, 1977, 163-198.

[6] Chuckrow, V.: On Schottky groups with applications to Kleinian groups. - Ann. of Math. 88, 1968, 47-61.

[7] EARLE, C.J.: Teichmüller theory. - In Discrete groups and automorphic functions, edited by W.J. Harvey, Academic Press, London, 1977, 143-162. 
[8] Earle, C.J., and J. Eells: A fibre bundle description of Teichmüller theory. - J. Differential Geom. 3, 1969, 16-43.

[9] Gardiner, F.P.: Teichmüller theory and quadratic differentials. - Wiley-Interscience, New York, 1987.

[10] Gentilesco, J.A.: Automorphisms of the deformation space of a Kleinian group. - Trans. Amer. Math. Soc. 248, 1979, 207-220.

[11] Hejhal, D.A.: On Schottky and Teichmüller spaces. - Adv. in Math. 15, 1975, 133-156.

[12] KRA, I.: Canonical mappings between Teichmüller spaces. - Bull. Amer. Math. Soc. 4, 1981, 143-179.

[13] KRA, I., and B. MAskit: The deformation space of a Kleinian group. - Amer. J. Math. 103, 1981, 1065-1102.

[14] LufT, E.: Actions of the homeotopy group of an orientable 3-dimensional handlebody. Math. Ann. 234, 1978, 279-292.

[15] Magnus, W., A. Karrass, and D. Solitar: Combinatiorial group theory. - WileyInterscience, New York, 1966.

[16] Maskit, B.: Self-maps on Kleinian groups. - Amer. J. Math. 93, 1971, 840-856.

[17] Maskit, B.: Kleinian groups. - Grundlagen der mathematischen Wissenschaften 287. Springer-Verlag, Berlin-Heidelberg-New York, 1988.

[18] Masur, H.: Measured foliations and handlebodies. - Ergodic Theory Dynamical Systems 6, 1986, 99-116.

[19] NAG, S.: The complex analytic theory of Teichmüller spaces. - Wiley-Interscience, New York, 1988.

Cornell University

Department of Mathematics

Ithaca, NY 14853

U.S.A.

Received 22 February 1991 